\title{
Lösungsmöglichkeiten nach fehlgeschlagenen Osteosynthesen: das Scaphoid
}

\author{
Jörg Schmidt, Dirk Calmez, Michael Fuhrmann
}

\section{Zusammenfassung}

Die Versorgung einer Scaphoidfraktur hat verschiedene Fehlermöglichkeiten. Neben den diagnostischen Schwierigkeiten und den indikatorischen Problemen können bei der Primärversorgung Probleme entstehen, die mit Substanzverlusten des Scaphoids einhergehen. Alle Rettungsverfahren haben das Problem der auf- wändigen Operationsverfahren und der längerfristigen Ruhigstellung. Bei länger bestehenden unverheilten Scaphoidfrakturen wird die Indikation zum operativen Vorgehen aufgrund der sicher zu erwartenden Handgelenkarthrose großzügig gestellt. Hier müssen die Verfahren jedoch individuell abgewogen und je nach Vorbefunden durchgeführt werden.

\section{Einleitung}

Der Bruch des Kahnbeins ist eine relativ seltene Fraktur. Nur 1-4\% aller Knochenbrüche beim Menschen machen eine Fraktur dieses kleinen Knochens des Karpus aus. Sieht man jedoch nur die Handwurzel, so liegt ein Kahnbeinbruch in etwa 35\% aller Frakturen vor. Leider werden auch noch heute die Verletzungen des Karpus unterschätzt und manche Scaphoidfraktur als „Handgelenkdistorsion" behandelt und übersehen, mit entsprechenden oftmals deletären Folgen für das Handgelenk $[3,5,19]$. Auch im Rahmen einer distalen Radiusfraktur kann eine ipsilaterale Scaphoidfraktur auftreten. Sofern man nicht darauf achtet, werden auch solche Frakturen übersehen und gelten in der Zeit der funktionellen Nachbehandlung der Radiusfraktur als unbehandelt [8].

Bis vor wenigen Jahren wurde die Behandlung der Kahnbeinbrüche als ein in der Hauptsache konservatives Problem

OP-JOURNAL 2004; 20: 116-120

(c) Georg Thieme Verlag KG Stuttgart · New York angesehen, so wie es bereits Böhler beschrieben hat. Er sah eine ununterbrochene Ruhigstellung in einem ungepolsterten Gips für in der Regel 6 Wochen indiziert, sollte dann keine Heilung eingetreten sein, war eine Ruhigstellung bis zu 16 Wochen angezeigt.

Solch lange Gipsruhigstellungen haben natürlich erhebliche Probleme und Mängel. So muss zum Beispiel bei einem wenig gepolsterten, gut anmodellierten Gips immer auf Druckschäden geachtet werden, Beschädigungen des Gipses müssen rechtzeitig erkannt und ausgebessert werden, da Unruhe im Gips mit Sicherheit zu einem Nichtdurchbau der Fraktur führen wird. Kontrollröntgenaufnahmen im Verlauf sind aufgrund der Gipsüberlagerung und der oftmals nur feinen Frakturlinie des Skaphoids kaum zu sehen. Auch sind Frakturen des proximalen Drittels des Skaphoids oftmals im Rahmen einer konservativen Therapie nicht zur Ausheilung zu bringen [17,18,22].

Neben der mittlerweile unbestrittenen größeren Sicherheit der operativen Behandlung in Bezug auf den Durchbau, insbesondere bei kritischen proximalen Frakturen, ist der Patientenkomfort, d.h. die nicht mehr notwendige Gipsruhigstellung bei freier Beweglichkeit der oberen Extremität, in den letzten Jahren so zunehmend in den Vordergrund getreten, dass der Anteil der operativ versorgten Kahnbeinbrüche ständig ansteigt. Als weiterer großer Vorteil ist zu werten, dass körperlich nicht belastete Patienten mit einem Kahnbeinbruch viel früher als bei der konservativen Behandlung mit all ihren Problemen wieder dem Arbeitsleben zugeführt werden können. Nicht zuletzt aus diesem Grund stellt die operative Behandlung auch der unkomplizierten Kahnbeinbrüche eine Alternative dar, die einem solchen Verletzten angeboten werden muss.

Die operative Versorgung birgt jedoch auch Probleme. Infektkomplikationen sind sehr selten. Häufiger ist jedoch die Schraubenfehllage bei der Versorgung mit der Doppelgewindeschraube oder auch immer noch die nicht angemessene Versorgung. Hier muss man die frühzeitige Revision von den Rettungsoperationen bei länger bestehenden nichtverheilten Scaphoiden unterscheiden [6].

Eine lange Ruhigstellung eines Handgelenkes sollte vermieden werden!

\section{Diagnostik}

Der Standard bei Verletzungen des Handgelenkes stellt die auf das Handgelenk zentrierte Röntgenaufnahme in 2 Ebenen dar, wobei neben der a.-p. Aufnahme eine halbschräge Aufnahme angefertigt wird. Ein zusätzlich streng seitlich eingestelltes Röntgenbild wird selten angefordert. Bei dem klinischen Verdacht ist die so genannte „Navicular-Serie“ (korrekterweise Scaphoid-Serie) der Standard. Besser sollte jedoch eine „Stecheraufnahme“ angefertigt werden. Hierbei wird der Zentralstrahl auf das Scaphoid eingestellt, die Hand zur Faust geballt und ellenseitig abgewinkelt. In dieser Position stellt sich das Scaphoid in ganzer Länge dar und kann so beurteilt werden $[1,2,9,16]$. 


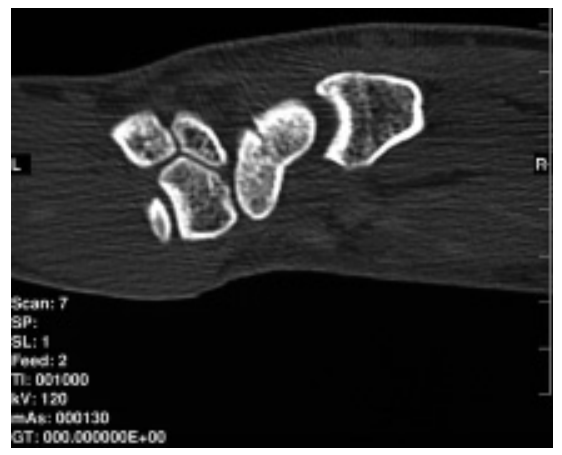

Abb.1 CT-Darstellung einer Scaphoidfraktur. Nur in dieser Technik sind die Abkippung, der Frakturspalt und die Struktur des Scaphoides genau zu beurteilen.

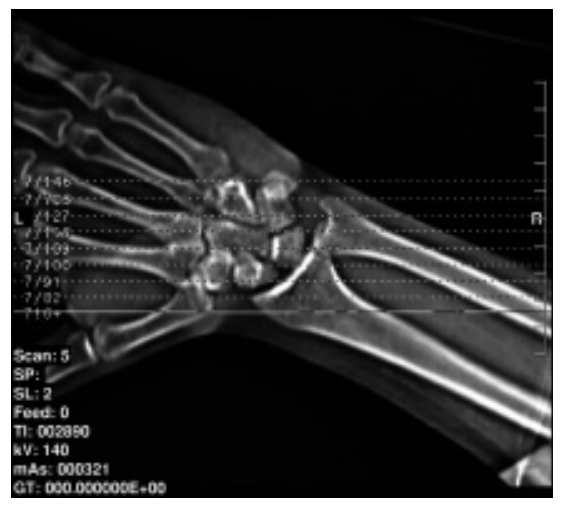

Abb.2 Auf eine Scaphoidparallele ist auf die Schichtung bei der CT-Untersuchung zu achten!

Sollte sich in der korrekt durchgeführten Primärdiagnostik der Verdacht auf eine Scaphoidfraktur weder bestätigen noch ausschließen lassen, kann man diese Untersuchungen nach 10 Tagen wiederholen, nachdem eine gewisse Resorption im Frakturspalt diesen besser zur Darstellung kommen lässt. Besser und auch für eine Planung der therapeutischen Maßnahmen aussagefähiger ist jedoch eine zeitnah durchgeführte CT-Untersuchung (Abb.1). Hier ist darauf zu achten, dass eine solche Untersuchung in Feinschichttechnik scaphoidparallel durchgeführt wird (Abb. 2). Dieser Untersuchungsalgorithmus stellt eine Scaphoidfraktur mit an Sicherheit grenzender Wahrscheinlichkeit dar.

Diagnostischer Algorithmus bei

Verdacht auf frische Kahnbeinfraktur:

- Handgelenk in zwei Ebenen

- Stecheraufnahme

- CT scaphoidparallel in Feinschichttechnik

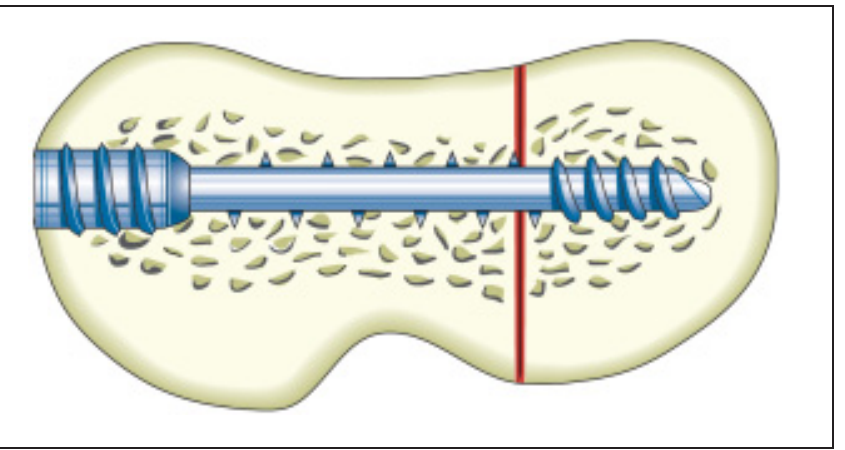

Abb. 3 Schema einer Doppelgewindeschraube.

\section{Prinzip der Erstversorgung}

Neben der immer noch bei bestimmten Frakturen und bei Patientenwunsch indizierten konservativen Behandlung ist die minimalinvasive von dorsal oder besser palmar durchgeführte Verschraubung heute die Versorgung der Wahl [21]. Sollte bei der präoperativen CT-Untersuchung eine Fragmentinterposition oder eine grobe Achsfehlstellung des Scaphoids dargestellt werden, muss eine von palmar durchgeführte offene Reposition und ebenfalls Verschraubung mit einer Doppelgewindeschraube erfolgen (Abb.3).

Insbesondere bei den perkutanen Verfahren muss die Lage der Schraube sehr selbstkritisch beurteilt werden. Eine postoperativ durchgeführte CT-Untersuchung kann durchaus eine Schraubenfehllage zeigen, die man intraoperativ nicht erkannt hat!

\section{Frühzeitige Rettungsverfahren}

\section{Nach konservativem Behandlungsversuch}

Sollte bei der konservativen Behandlung eine Scaphoidfraktur nach 9 Wochen nicht zu einen ausreichenden Durchbau gekommen sein, das heißt im KontrollCT in scaphoidparalleler Einstellung und Feinschichttechnik in 3 aufeinander folgenden Schichten keine Durchbauzeichen haben, muss dem Patienten eine operative Revision zwingend angeboten werden [11]. Es muss abgewogen werden, ob eine einfache perkutane Verschraubung ausreichend ist oder ob eine Spongiosaplastik im Sinne einer modifizierten Matti-Russe-Plastik erforderlich wird. Eine Entscheidungshilfe hierbei ist die MRT-Untersuchung, die uns eine Aussage über die Vitalität und Durchblutung der beiden Fragmente bringt.

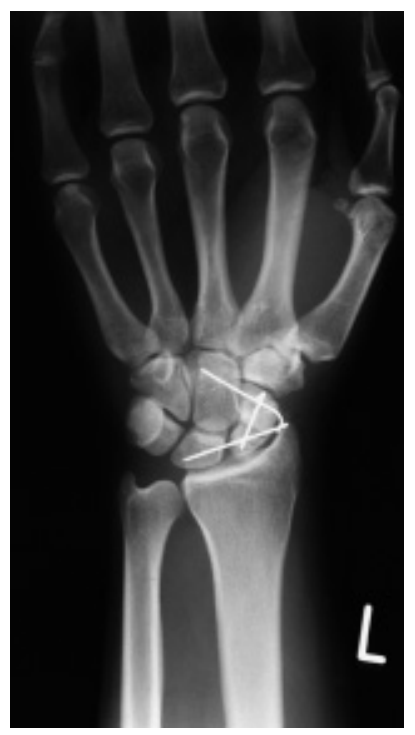

Abb.4 Die Versorgung einer Scaphoidfraktur mit KirschnerDrähten ist nicht korrekt!

\section{Nach operativer Versorgung}

Gleich ob eine Verschraubung oder eine andere, nicht sachgerechte Versorgung (Abb.4) der Fraktur vorgenommen wurde, ergibt sich das große Problem des Substanzverlustes im Scaphoid [6,15]. Bei der Verschraubung ist in der Regel die Schraube aus einem der Fragmente herausgebrochen oder, und dies zumeist, in den radiokarpalen Gelenkspalt ausgetreten (Abb.5). In der Regel kommt es dabei zu einer Zunahme des intrascaphoidalen Winkels und einer Rotationsfehlstellung des Kahnbeines. Radiologisch findet man Lysen um die Schraube als Zeichen des Substanzverlustes an Spongiosa im Knochen. Problematisch sind aber vor allem Ausbrüche der Spongiosa. Eine Verankerung von neuen Implantaten ist in der Regel nicht mehr möglich.

Nach einer offenen Reposition der Stellung hilft in einer solchen Situation eigentlich nur noch eine Implantation eines 


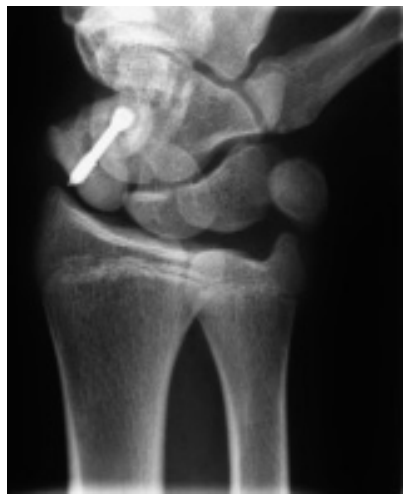

Abb. 5 Eine ausgerissene Doppelgewindeschraube hinterlässt große Substanzdefekte im Kahnbein!

kortikospongiösen Spanes im Sinne einer modifizierten Matti-Russe-Plastik. Dadurch kann in der Regel der Substanzverlust aufgefüllt werden, gleichzeitig wird die Stellung und Länge des Kahnbeines gehalten. Ob eine zusätzliche Sicherung mit Kirschner-Drähten notwendig wird, muss intraoperativ entschieden werden.

In wenigen ausgewählten Fällen mit ausgedehnten Zerstörungen des Kahnbeines kann eine radiale Teilarthrodese zwischen Scaphoid, Lunatum und Capitatum (SLC-Arthrodese) notwendig werden $[7,10]$. Unter Aufgabe eines Teiles der Beweglichkeit des Handgelenkes gewinnt man aber insbesondere bei jüngeren, handwerklich tätigen Patienten eine Steigerung der beschwerdereduzierten Belastbarkeit. Hierzu stehen seit einiger Zeit speziell geformte Implantate zur Verfügung.

Bei allen Verfahren sind jedoch längerfristige Ruhigstellungen von 6 bis 12 Wochen, je nach CT-gesichertem Durchbau, notwendig. Somit ist der Vorteil der primären operativen Versorgung, also die frühfunktionelle Behandlung vergeben. Arbeitsunfähigkeitszeiten bis zu 4 Monaten fallen an, in manchen Fällen ist auch ein Berufswechsel notwendig.

Bei frühzeitigen Revisionsoperationen ist mit Substanzdefekten zu rechnen. Daher ist eine Knochentransplantation meist nicht zu umgehen.

\section{Rettungsverfahren bei}

Scaphoidpseudarthrosen

Sollte nach einer Behandlungszeit von 4 bis 6 Monaten kein Durchbau einer Scaphoidfraktur erreicht werden, spricht man von einer Scaphoidpseudarthrose. Langzeituntersuchungen von Martini [19] konnten zeigen, dass nach einer Zeit von 15 Jahren in Handgelenken mit bestehenden Pseudarthrosen immer eine Handgelenkarthrose entsteht. Die Zeitverläufe sind immer gleich und dienen als Grundlage der Einteilung und Klassifikation der Handgelenkarthrosen. Da es sich um einen zunehmenden Kollaps des Handgelenkes handelt, wird dieser Zustand auch SNAC-Wrist (scaphoid non-union advanced collaps) genannt $[3,5]$. Aus der Konstanz der Abläufe resultiert auch die Forderung, dass Scaphoidpseudarthrosen dann behandelt werden müssen, wenn man sie erkennt und nicht erst wenn sie Beschwerden bereiten.

Diagnostische Voraussetzungen der Behandlung sind neben den Röntgenaufnahmen in zwei Ebenen und der streng seitlichen Aufnahme zur Beurteilung der intrakarpalen Winkelverhältnisse die Durchführung einer MRT-Untersuchung, vorteilhaft mit Kontrastmittel, zur Beurteilung der Vitalität der Scaphoidfragmente und die einer operativen Stabilisierung vorgeschalteten Arthroskopie des Handgelenkes zur Beurteilung der Knorpelverhältnisse. Die Art der operativen Stabilisierung ist anhand der Befunde dann individuell zu stellen, die wesentlichen Verfahren werden nachfolgend dargestellt.

Diagnostische Voraussetzungen der Rettungsoperationen:

- Röntgenaufnahmen Handgelenk in 2 Ebenen und streng seitlich

- MRT

- Handgelenksarthroskopie

\section{Matti-Russe-Plastik}

Es handelt sich wie schon beschrieben um die Längsimplantation eines kortikospongiösen Spanes über die Pseudarthrosezone nach Ausfräsen des Scaphoids. Der Vorteil ist insbesondere beim jungen Patienten darin zu sehen, dass vitalitätsgeminderte proximale Pole „reanimiert“ werden können. Nachteilig wirkt sich die lang andauernde Ruhigstellung des Handgelenkes von bis $\mathrm{zu} 12$ Wochen aus. Als Voraussetzung müssen allerdings in allen Anteilen des radiokarpalen Gelenkes gute Knorpelverhältnisse vorliegen (Abb. 6).

\section{Korrektur nach Fernandez-Fisk}

Bei dieser Methode wird von palmar eine Korrektur des intrakarpalen Winkels des

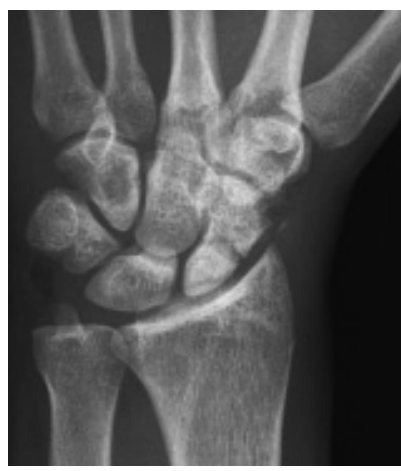

Abb.6 In der postoperativen Röntgenkontrolle nach einer Matti-Russe-Plastik ist der eingebrachte Span kaum zu erkennen.

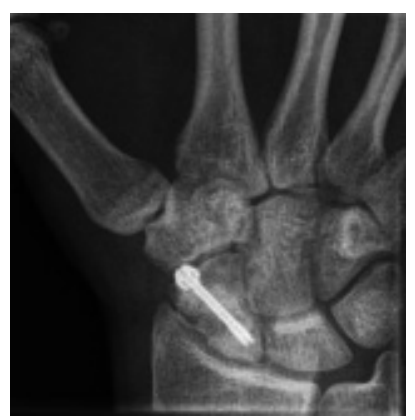

Abb. 7 Der aufrichtende Span bei der Fernandez-Fisk-Operation muss mit einer Schraube gehalten werden.

Scaphoides durch Resektion der Pseudarthrose und Einbolzung eines keilförmigen kortikospongiösen Spanes durchgeführt $[12,13]$. Zusätzlich ist bei dieser Operationsmethode eine Stabilisierung, vorzugsweise durch eine Doppelgewindeschraube durchzuführen, um den Span in seinem Bett zu halten (Abb. 7). Alternativ sind auch Kirschner-Drähte oder eine palmar angelegte Platte (z.B. die ENDER-Platte) möglich, die allerdings nach dem Durchbau zu entfernen sind. Der Vorteil dieses Verfahrens liegt in der sehr guten Korrekturmöglichkeit der Scaphoidstellung und damit der Handgelenkkinematik und sollte in allen Fällen in Erwägung gezogen werden. Nachteilig wirkt sich auch bei dieser Methode die Ruhigstellung von 6 Wochen aus und die alle Operationen der Scaphoidpseudarthrosen belastende Misserfolgsquote von ca. 30\%. Die Voraussetzungen für dieses Verfahren sind neben den arthroskopisch gesicherten intakten Knorpelverhältnissen auch im MRT gesicherte normale Vitalitätsverhältnisse beider Scaphoidanteile. 


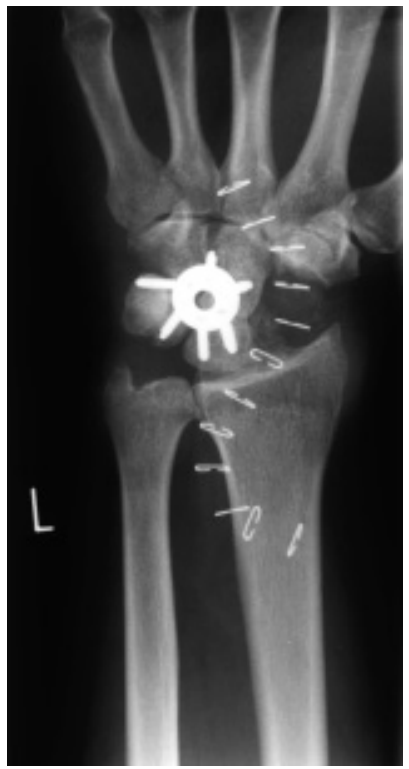

Abb. 8 Mediokarpale Teilarthrodese mit "Spider-Plate“ und ausgiebiger Spongiosaplastik.

\section{SLC-Arthrodese}

Diese Art der Teilarthrodesen kommt bei der Pseudarthrose nur selten zum Einsatz und bleibt der Frühintervention vorbehalten [7]. In dieser Art der Pseudarthrosenbehandlung bestehen keine Vorteile gegenüber den oben genannten und bleiben hier den zweiten Revisionen vorbehalten, falls eine der oben genannten Verfahren nicht zum Erfolg führt.

\section{Mediokarpale Teilarthrodese}

Dieses Rettungsverfahren findet seine Anwendung vor allem bei länger bestehenden Scaphoidpseudarthrosen der Klassifikation SNAC 2 und $3[25,26]$. Bei arthroskopisch gesicherten Knorpelschäden korrespondierend zum Kahnbein (Fossa scaphoides des Radius) ist in der Regel korrespondierend zum Mondbein (Fossa lunata des Radius) immer mit einem gut erhaltenen Knorpelüberzug zu rechnen. Daher beruht dieses Verfahren auf der Entfernung des Scaphoids und einer die Länge und Stellung der Karpalknochen erhaltenden Teilarthrodese der ulnaren Handwurzelknochen. Dazu werden die Gelenkspalte zwischen Lunatum, Capitatum, Triquetrum und Hamatum ausgefräst, mit Beckenkammspongiosa aufgefüllt und stabilisiert. Dazu stehen neuere Implantate zur Verfügung, die wegen ihres Aussehens „Spider-Plate“ genannt werden (Abb.8). Eine Beweglichkeit im Handgelenk wird zwischen Radi- us und Lunatum erhalten. Der Bewegungsumfang des Handgelenkes wird in der Regel auf einen Bewegungsumfang von $30^{\circ}-0-30^{\circ}$ eingeschränkt.

\section{Seltene Verfahren und Außenseitermethoden}

Bei höher gradigen Arthrosen des Handgelenkes bei erhaltenem Knorpelüberzug der Fossa lunata wird von manchen Autoren die Entfernung der proximalen Handwurzelreihe (proximal row carpectomy, PRC) bevorzugt [10]. Durch den Höhenverlust der Handwurzel kommt es jedoch zu Kraftverlusten. Damit hat dieses Verfahren in unseren Augen einen Nachteil gegenüber der mediokarpalen Teilarthrodese.

Bei durchblutungsgeminderten Scaphoidfragmenten oder aber auch bei Revisionsoperationen nach der Versorgung einer Scaphoidpseudarthrose kommt der vaskularisierte, gefäßgestielte Span zur Anwendung [23,24,27]. Bei diesen Rettungsverfahren werden Knochenblöcke an ihrem ernährenden Gefäßstiel in das Scaphoid geschwenkt.

Als Außenseitermethode ist die Anwendung von niedrig gepulstem Ultraschall zu nennen [20]. Durch die Ultraschallapplikation sollen Pseudarthrosen auch an anderen Knochen zum Ausheilen gebracht werden können.

Über die Art des Rettungsverfahrens muss individuell nach den Vorbefunden entschieden werden!

\section{Schlussfolgerung}

Die primäre operative Versorgung einer Scaphoidfraktur hat für den Patienten Vorteile gegenüber dem konservativen Vorgehen. Sollte eine solche Versorgung jedoch misslingen, müssen zum Teil sehr aufwändige Rettungsoperationen durchgeführt werden. Mit einem solchen Eingriff jedoch darf nicht gezögert werden, damit die unangenehme Komplikation der Handgelenkarthrose möglichst umgangen werden kann. Die individuelle Indikation des Verfahrens sollte von Erfahrenen gestellt werden.

\section{Literatur}

${ }^{1}$ Annamalai G, Raby N. Scaphoid and pronator fat stripes are unreliable soft tissue signs in the detection of radiographically occult fractures. Clin. Radiol 2003; 58: 798 - 800

${ }^{2}$ Bain GI, Bennett JD, Richards RS, Slethaug GP, Roth JH. Longitudinal computed tomography of the scaphoid: a new technique. Skeletal Radiol 1995; 24: $271-273$

${ }^{3}$ Ballas MT, Tytko J, Mannarino F. Commonly missed orthopedic problems. Am Fam Physician 1998; 57: 267-274

${ }^{4}$ Berdia S, Wolfe SW. Effects of scaphoid fractures on the biomechanics of the wrist. Hand Clin 2001; 17: 533

5 Beutel FK, Wilhelm K. [Relation of necrosis to outcome and site of fracture in scaphoid pseudarthrosis]. Handchir Mikrochir Plast Chir 1999; 31: 207-211

${ }^{6}$ Bickert B, Baumeister S, Sauerbier M, Germann G. Use of a cannulated $3.0 \mathrm{~mm} \mathrm{AO}$ screw with an intraosseous support washer in osteosynthesis of the scaphoid: results and analysis of problems in 28 cases. Handchir Mikrochir Plast Chir 2000; 32: 277 - 282

Calandruccio JH, Gelberman RH, Duncan SF, Goldfarb CA, Pae R, Gramig W. Capitolunate arthrodesis with scaphoid and triquetrum excision. J Hand Surg [Am.]. 2000; 25: $824-832$

${ }^{8}$ Chang CH, Tsai YS, Sun JS, Hou SM. Ipsilateral distal radius and scaphoid fractures. J Formos Med Assoc 2000; 99: 733-737

${ }^{9}$ Compson JP. The anatomy of acute scaphoid fractures: a three-dimensional analysis of patterns. J Bone Joint Surg $\mathrm{Br}$ 1998; 80: $218-224$

${ }^{10}$ Culp RW, Williams CS. Proximal row carpectomy for the treatment of scaphoid nonunion. Hand Clin 2001; 17: 663-669

${ }^{11}$ Dias JJ. Definition of union after acute fracture and surgery for fracture nonunion of the scaphoid. J Hand Surg [Br.] 2001; 26: $321-325$

${ }^{12}$ Fernandez DL, Eggli S. Scaphoid nonunion and malunion. How to correct deformity. Hand Clin 2001; 17: 631-646

${ }^{13}$ Garcia-Lopez A, Perez-Ubeda MJ, Marco F, Molina M, Lopez-Duran L. A modified technique of four-bone fusion for advanced carpal collapse (SLAC/SNAC wrist). J Hand Surg [Br.] 2001; 26: 352 - 354

${ }^{14}$ Hakimi M, Linhart W, Windolf J. Functional results two years after the treatment of a delayed diagnosed scapho-capitate fracture syndrome. Handchir Mikrochir Plast Chir 2003; 35: $338-341$

${ }^{15}$ Inoue G, Kuwahata Y. Repeat screw stabilization with bone grafting after a failed Herbert screw fixation for acute scaphoid fractures and nonunions. J Hand Surg [Am.]. 1997; 22: $413-418$

${ }^{16}$ Jacobsen S, Hassani G, Hansen D, Christensen O. Suspected scaphoid fractures. Can we avoid overkill? Acta Orthop Belg 1995 61: $74-78$

${ }^{17}$ Jupiter JB, Shin AY, Trumble TE, Fernandez DL. Traumatic and reconstructive problems of the scaphoid. Instr Course Lect 2001; 50: $105-22$

${ }^{18}$ Kozin SH. Incidence, mechanism, and natural history of scaphoid fractures. Hand Clin 2001; 17: 515-524

${ }^{19}$ Martini AK. Der Spontanverlauf der Kahnbeinpseudarthrose. Orthopäde 1994; 23 : 249-254

${ }^{20}$ Mayr E, Rudzki MM, Rudzki M, Borchardt B, Hausser H, Ruter A. Does low intensity, puls- 
ed ultrasound speed healing of scaphoid fractures?. Handchir Mikrochir Plast Chir 2000; 32: 115-122

${ }^{21}$ Polsky MB, Kozin SH, Porter ST, Thoder JJ. Scaphoid fractures: dorsal versus volar approach. Orthopedics 2002; 25: 817-819

22 Roolker W, Maas M, Broekhuizen AH. Diagnosis and treatment of scaphoid fractures, can non-union be prevented? Arch Orthop Trauma Surg 1999; 119: 428-431

${ }^{23}$ Shin AY, Bishop AT. Vascularized bone grafts for scaphoid nonunions and kienbock's disease. Orthop Clin North Am 2001; 32: 263 277
${ }^{24}$ Steinmann SP, Bishop AT. A vascularized bone graft for repair of scaphoid nonunion. Hand Clin 2001; 17: 647-653

${ }^{25}$ Tomaino MM. Intercarpal fusion for the treatment of scaphoid nonunion. Hand Clin 2001; 17: 671 -686

${ }^{26}$ Tunnerhoff HG, Das GK, Haussmann P. Functional results of medio-carpal partial arthrodesis with excision of the scaphoid. Handchir Mikrochir Plast Chir 2001; 33: $408-417$

${ }^{27}$ Uerpairojkit C, Leechavengvongs S, Witoonchart K. Primary vascularized distal radius bone graft for nonunion of the scaphoid. J Hand Surg [Br.] 2000; 25: 266-270
Dr. med Jörg Schmidt

Chefarzt

Dr. med. Dirk Calmez

Facharzt

Dr. med. Michael Fuhrmann

Oberarzt, Bereichsleiter Handchirurgie

Klinik für Unfall-, Hand- und Wiederherstellungschirurgie

im HELIOS Klinikum Berlin

Hobrechtsfelder Chaussee 100

D-13125 Berlin 